Original Research Paper

\title{
Diosgenin Biosynthesis in the Sprouts of Fenugreek as Influenced by Chitosan
}

\author{
${ }^{1}$ Do Yeon Kwon, ${ }^{2}$ Jae Kwang Kim and ${ }^{3}$ Sang Un Park \\ ${ }^{I}$ Smart Farm Research Center, KIST Gangneung Institute of Natural Products, \\ 679, Saimdang-ro, Gangneung, Gangwon-do 25451, Korea \\ ${ }^{2}$ Division of Life Sciences and Convergence Research Center for Insect Vectors, \\ Incheon National University, Incheon 22012, Korea \\ ${ }^{3}$ Department of Crop Science, Chungnam National University, 99 Daehak-Ro, Yuseong-Gu, Daejeon 34134, Korea
}

\section{Article history}

Received: 29-12-2018

Revised: 06-03-2019

Accepted: 02-04-2019

Corresponding Autor:

Jae Kwang Kim

Division of Life Sciences and

Convergence Research

Center for Insect Vectors,

Incheon National University,

Incheon 22012, Korea

Tel: $+82-32-835-8241$

Fax: $+82-32-835-0763$

Email:kjkpj@inu.ac.kr

Sang Un Park

Department of Crop Science,

Chungnam National

University, 99 Daehak-Ro,

Yuseong-Gu, Daejeon 34134,

Korea

Tel: $+82-42-821-5730$

Fax: $+82-42-822-2631$

Email: supark@cnu.ac.kr

\begin{abstract}
Trigonella foenum-graecum L. (Fabaceae), known as Fenugreekis an annual herb, contains various secondary metabolites including steroidal saponins, flavonoids, phenolic compounds and alkaloids. This study was planned to investigate the effect of concentrations of chitosan on growth, expression of diosgenin biosynthetic genes and diosgenin accumulation in sprouts of $T$. foenum-graecum. Results revealed that there is no positive response of chitosan in any of the studied growth parameters i.e. shoot and root length, fresh and dry weight. In control treatment without any chitosan solution the highest values for all the parameters were observed. The results indicated that increasing concentrations of chitosan had no significant effect on shoot and root lengths. The fresh weight of sprouts treated with chitosan decreased markedly, but not significantly, compared with that of the control. However, the dry weight of sprouts treated with chitosan was almost similar to that of the control. Diosgenin biosynthetic pathway in response to different concentration of chitosan enhanced to an increasing level in the expression of all treated sprouts than those of no chitosan (control) treated sprouts. The highest expression level was detected when treated with $0.05 \mathrm{mg} / \mathrm{mL}$ chitosan in most of the genes except TfSQS. The expression of TfSQS was slightly higher in sprouts treated with $0.01 \mathrm{mg} / \mathrm{mL}$ chitosan than in those treated with $0.05 \mathrm{mg} / \mathrm{mL}$ chitosan. The highest expression level was in $T f C A S$ at $0.05 \mathrm{mg} / \mathrm{mL}$ chitosan exhibiting 3 times higher expressing than that of control. The increasing expression trend for both TfSQLE and TfSTRL remained up to $0.05 \mathrm{mg} / \mathrm{mL}$ chitosan showing 1.7 times more expression in this concentration and then started to decrease the expression level. Diosgenin content in the T. foenum-graecum sprouts did not respond positively to chitosan treatment. Our findings could be potentially implemented for future studies in this arena.
\end{abstract}

Keywords: Chitosan, Diosgenin, Fenugreek Sprouts, Gene Expression

\section{Introduction}

Genus Trigonella includes 75 species. The name comes from the Latin Trigonus, which means "threeangled", in reference to the small, triangular appearance of the flower (Christen, 2002). This is known as Fenugreek, Trigonella foenum-graecum L. (Fabaceae), is an annual herb (Snehlata and Payal, 2012) cultivated widely from Iran to Northern India, as well as in Egypt, Southern Europe, China, West Africa, Pakistan and the Mediterranean countries.
One of the saponins present in fenugreek is diosgenin, a major bioactive chemical compound structurally similar to cholesterol and other steroids (Rahmati-Yamchi et al., 2014). In the pharmaceutical industry, diosgenin is the principal precursor compound in the manufacture of several synthetic steroidal drugs (Chen et al., 2015). It also represents a promising bioactive biomolecule that exhibits various biological properties; these include hypolipidemic, hypoglycemic, antioxidant, anti-inflammatory and antiproliferative activities (Jesus et al., 2016). 
Chitosan is a chitin derivative, a biopolymer characterized by unique properties, such as bioactivity, biocompatibility and non-allergenicity (Salachna and Zawadzińska, 2014; Malerba and Cerana, 2016). Chitosan acts in controlling plant pathogens, enhancing germination index, reducing germination time, increasing shoot height, root length, shoot and root dry weights (El Hadrami et al., 2010) and eliciting natural defense response mechanisms (Walker et al., 2004).

In this study, we investigated diosgenin biosynthesisrelated gene expression and diosgenin content in fenugreek sprouts with chitosan treatment.

\section{Materials and Methods}

\section{Plant materials}

Seeds of $T$. foenum-graecum were purchased from Aram Seed Company, Seoul, Korea and were kept at $4{ }^{\circ} \mathrm{C}$ until use. Fifty seeds were sown in each pot having top diameter $116 \mathrm{~mm}$, height $105 \mathrm{~mm}$, bottom diameter $85.5 \mathrm{~mm}$ which was filled with vermiculite and placed in a growth chamber under a $16 \mathrm{~h}$ photoperiod $(300$ $\left.\mu \mathrm{mol} / \mathrm{m}^{2} \mathrm{~s}\right)$ at $25^{\circ} \mathrm{C}$.

\section{Chitosan Treatment of Fenugreek Sprouts}

A stock solution of chitosan (Sigma-Aldrich) was prepared by addition $10 \mathrm{~g}$ chitosan to $1 \mathrm{~L}$ of $1 \%$ acetic acid $(0.1 \mathrm{M})$ and then the mixture was heated to $40-50^{\circ} \mathrm{C}$ with continuous stirring, for a period of $24 \mathrm{hrs}$. Different diluted concentration was prepared according to experimental specification $(0.1,0.5$ and $1 \mathrm{mg} / \mathrm{ml})$ from the chitosan stock solution. Same amount of these solutions $(0.1,0.5$ and $1 \mathrm{mg} / \mathrm{ml})$ were applied daily up to the time before sampling. All fenugreek sprouts were harvested at 10 days after chitosan treatment. The data were recorded on the following parameters i.e., shoot and root lengths and their fresh weight. The sprouts were frozen in liquid nitrogen and stored at $-80^{\circ} \mathrm{C}$ immediately after collection until used for analysis.

\section{$R N A$ Isolation and cDNA Synthesis}

For RNA isolation and cDNA synthesis, samples were grinded using liquid nitrogen and made them into a fine powder and then moved to $1.5 \mathrm{~mL}$ micro-centrifuge tubes. Total RNA was separated from the frozen tissues using a Plant Total Mini Kit (Generaid Taiwan) following the protocol described in the manufacturer instructions. After completion of the first step, Trizol was used to homogenize cells; and then chloroform was inserted for phase separation and then added cold isopropanol for RNA precipitation. Purification and total RNA concentration was measured at $260.280 \mathrm{~nm}$ (NanoVue Plus Spectrophotometer, GE Healthcare BioScience Crop, USA) and RNA integrity was proved on $1 \%$ formaldehyde RNA agarose gels. One $\mu \mathrm{g}$ of total
RNA was used for cDNA synthesis. This process was done using a ReverTra Ace- $\alpha$ kit (Toyobo, Japan) for reverse-transcription and then used oligo $(\mathrm{dT})_{20}$ primer with a 20 -fold dilution of the $20-\mu \mathrm{L}$ resulting cDNA products as template for real time-PCR analysis.

\section{Real-Time PCR}

Here in this study qRT-PCR was performed in a BIORAD CFX96 Real-time PCR system (Bio-Rad Laboratories, USA). The primers Squalene Synthase (SQS), Squalene monooxygenase (SQLE) and cycloartenol synthase $(C A S)$ were designed using Primer 3 (http://bioinfo.ut.ee/primer3-0.4.0/). Sterol-3-ßglucosyl transferase $(S T R L)$ was arranged from Bioneer, according to transcriptome data of Gujarat Methi-1 variety published (Chaudhary et al., 2015). For housekeeping gene, the $18 \mathrm{~S}$ gene was used in this study. The PCR products amplified from cDNA were purified by using a Gel Extraction Kit (ELPIS Biotech, Korea) which was used for quantification of the standard and finally the concentration of the products was determined to calculate the number of cDNA copies. The SYBR Green qRT-PCR assay was done in a total volume of 20 $\mu \mathrm{L}$ containing $10 \mu \mathrm{L}$ of $2 \times$ Real-Time PCR Smart mix (Biofact Korea), $1 \mu \mathrm{L}(10 \mathrm{pmol} / \mu \mathrm{L})$ of each specific primer, $5 \mu \mathrm{L}$ of template cDNA and $3 \mu \mathrm{L}$ of D.W. The following conditions as follows: $15 \mathrm{~s}$ pre-denaturation at $95^{\circ} \mathrm{C}$, followed by $95^{\circ} \mathrm{C}$ for $20 \mathrm{~s}$; annealing at $55^{\circ} \mathrm{C}$ for $40 \mathrm{~s}$ and 40 cycles of elongation at $72^{\circ} \mathrm{C}$ for $20 \mathrm{~s}$ were maintained. Final extension happened at $72^{\circ} \mathrm{C}$ for 10 min. The experiments were repeated thrice and the results were represented by means $\pm \mathrm{SD}$.

\section{High Performance Liquid Chromatography Analysis}

For the detection of diosgenin, samples were dried in a freeze-dryer at $80^{\circ} \mathrm{C}$ for about $48 \mathrm{~h}$. An amount of 100 $\mathrm{mg}$ dried samples were incubated in $5 \mathrm{~mL}$ of $20 \% \mathrm{H}_{2} \mathrm{SO}_{4}$ in $70 \%$ isopropanol, under water-bath for $8 \mathrm{~h}$ at $80^{\circ} \mathrm{C}$. After that, samples were vortexed every $30 \mathrm{~min}$ during incubation. Extracts were centrifuged at $12,000 \mathrm{rpm}$ at $4^{\circ} \mathrm{C}$ for $10 \mathrm{~min}$. After centrifugation, the supernatants were moved to a new $15 \mathrm{~mL}$ conical tube and added with $5 \mathrm{~mL}$ n-hexane, prior to overtaxing and strong shaking. Next, they were centrifuged at $12,000 \mathrm{rpm}$ at $4{ }^{\circ} \mathrm{C}$ for 10 min. Only supernatants were moved to new $15 \mathrm{~mL}$ conical tubes. For maximum extraction this step was repeated three times. By using a Rotovac evaporator extracts were dried by evaporating the solvent at $40^{\circ} \mathrm{C}$ in. After that dried crude extracts were solubilized in 1 $\mathrm{mL}$ HPLC acetonitrile and filtered using $0.45 \mu \mathrm{m}$ PTFE hydrophilic syringe filter (Advantec DISMIC-13HP, Toyo Roshi Kaisha, Ltd., Tokyo, Japan) for High Performance Liquid Chromatography (HPLC) analysis. The desired compound diosgenin was isolated on an 
Agilent 1100 series HPLC system with a $C_{18}$ reverse phase column $(250 \times 4.6 \mathrm{~mm}, 5 \mu \mathrm{m}$; RStech, Daejeon, Korea) following retention time and detected with a UV detector at $203 \mathrm{~nm}$. Mobile phase was consisted of water as Solvent A and solvent B consisted of acetonitrile. The retention time for diosgenin was $20 \mathrm{~min}$. The initial proportion of the mix was $10 \%$ solvent $\mathrm{A}$ and $90 \%$ acetonitrile. The flow rate of the solvent was maintained at $1.0 \mathrm{ml} / \mathrm{min}$. Diosgenin was detected by matching the retention time and spectral characteristics to those from the single HPLC run of a known diosgenin standard.

\section{Statistical Analysis}

All experiments and samples were done thrice for each treatment. The data were analyzed using IBM SPPS Statistic (v24) provides an application-oriented introduction to the statistical component of IBM ${ }^{\circledR}$ SPPS by Tukey's Multiple Range Test., using one-way ANOVA at the 5\% significance level.

\section{Results and Discussion}

Effects of Chitosan Treatments on Growth, Diosgenin Biosyntheticgene Expression and Diosgenincontent in the Sprouts of T. foenum-graecum

The effects of different concentrations of chitosan $(0.01,0.05$ and $0.1 \mathrm{mg} / \mathrm{mL})$ on the growth of 10-day old T. foenum-graecum sprouts are presented in Fig. 1. The results indicated that, there is no positive response of chitosan in any of the studied growth parameters i.e., shoot and root length, fresh and dry weight. In control treatment without any chitosan solution the highest values for all the parameters were observed. The results indicated that increasing concentrations of chitosan had no significant effect on shoot and root lengths. The fresh weight of sprouts treated with chitosan decreased markedly, but not significantly, compared with that of the control. However, the dry weight of sprouts treated with chitosan was almost similar to that of the control. Growth of root in terms of root length was suppressed greatly than that of shoot length. Here it was observed that the highest root length of $7.9 \mathrm{~cm}$ which was observed in control treatment whereas this root length reached to $6.0 \mathrm{~cm}$ at the time of application of the highest concentration of chitosan $(0.1 \mathrm{mg} / \mathrm{ml})$. On the other hand shoot length did not vary sharply as like as root length. In this case, the highest shoot length of 3.9 $\mathrm{cm}$ which was observed in control treatment whereas this shoot length reached to $3.6 \mathrm{~cm}$ at the time of application of the highest concentration of chitosan $(0.1 \mathrm{mg} / \mathrm{ml})$. In case of fresh weight it was around 1.3 $\mathrm{g}$ for the control treatment whereas this value goes below $0.7 \mathrm{~g}$ at the time of application of the highest concentration of chitosan $(0.1 \mathrm{mg} / \mathrm{ml})$. The variation in dry weight from control to the highest level of chitosan was almost similar.

The expression of all genes related to the diosgenin biosynthetic pathway in response to different concentration of chitosan enhanced to an increasing level in the expression of all treated sprouts than those of no chitosan (control) treated sprouts (Fig. 2). The highest expression level was detected when treated with 0.05 $\mathrm{mg} / \mathrm{mL}$ chitosan in most of the genes except TfSQS. The expression of TfSQS was slightly higher in sprouts treated with $0.01 \mathrm{mg} / \mathrm{mL}$ chitosan than in those treated with $0.05 \mathrm{mg} / \mathrm{mL}$ chitosan. The expression of $S Q S$ was higher in $0.01 \mathrm{mg} / \mathrm{mL}$ chitosan treated sprouts than in the control, but decreased considerably with an increase in chitosan concentration. The highest expression level was in $T f C A S$ at $0.05 \mathrm{mg} / \mathrm{mL}$ chitosan exhibiting 3 times higher expressing than that of control. The increasing expression trend for both TfSQLE and TfSTRL remained up to $0.05 \mathrm{mg} / \mathrm{mL}$ chitosan showing 1.7 times more expression in this concentration and then started to decrease the expression level.

Diosgenin content in the T. foenum-graecum sprouts did not respond positive to chitosan treatment (Fig. 3). The diosgenin content was higher in control treatment than any one of the chitosan treatment. It is notated that chitosan treatment suppressed the accumulation of diosgenin content.

It is observable that with no chitosan treatment showed the positive response for the growth parameters like shoot and root length and their fresh and dry weight. The highest values for all the parameters were observed in control treatment without any chitosan treatment But due to the treatment of chitosan the diosgenin biosynthetic genes i.e., TfSQS, TfSQLE, TfCAS and TfSTRL were upregulated. In relation with our studied some previous research findings have shown that chitosan enhanced to accumulate higher amount of secondary metabolites. In a previous study reported that chitosan enhanced to accumulate sorgoleone in sorghum roots (Uddin et al., 2010). Chitosan belongs to a family of biopolymers naturally and industrially obtained by $N$ deacetylation of chitin, which is almost never complete (Ferri and Tassoni, 2011). Chitosan treatment increased the total weight by $12.9 \%$ and the germination rate by $16 \%$ compared to those of the control in sunflower sprouts (Cho et al., 2008). Further, with an increase in chitosan concentration from 2 to $8 \mathrm{mg} / \mathrm{mL}$, the phenolic acid accumulation increased, such as trans-cinnamic acid, benzoic acid, caffeic acid, ferulic acid and sinapic acid (Reddy et al., 1999). No correlation was found between gene expression and chitosan concentration in fenugreek sprouts. These results indicated that, although chitosan concentration and gene expression varied in sprouts, chitosan treatment was not a viable option for increasing diosgenin content in fenugreek sprouts. 


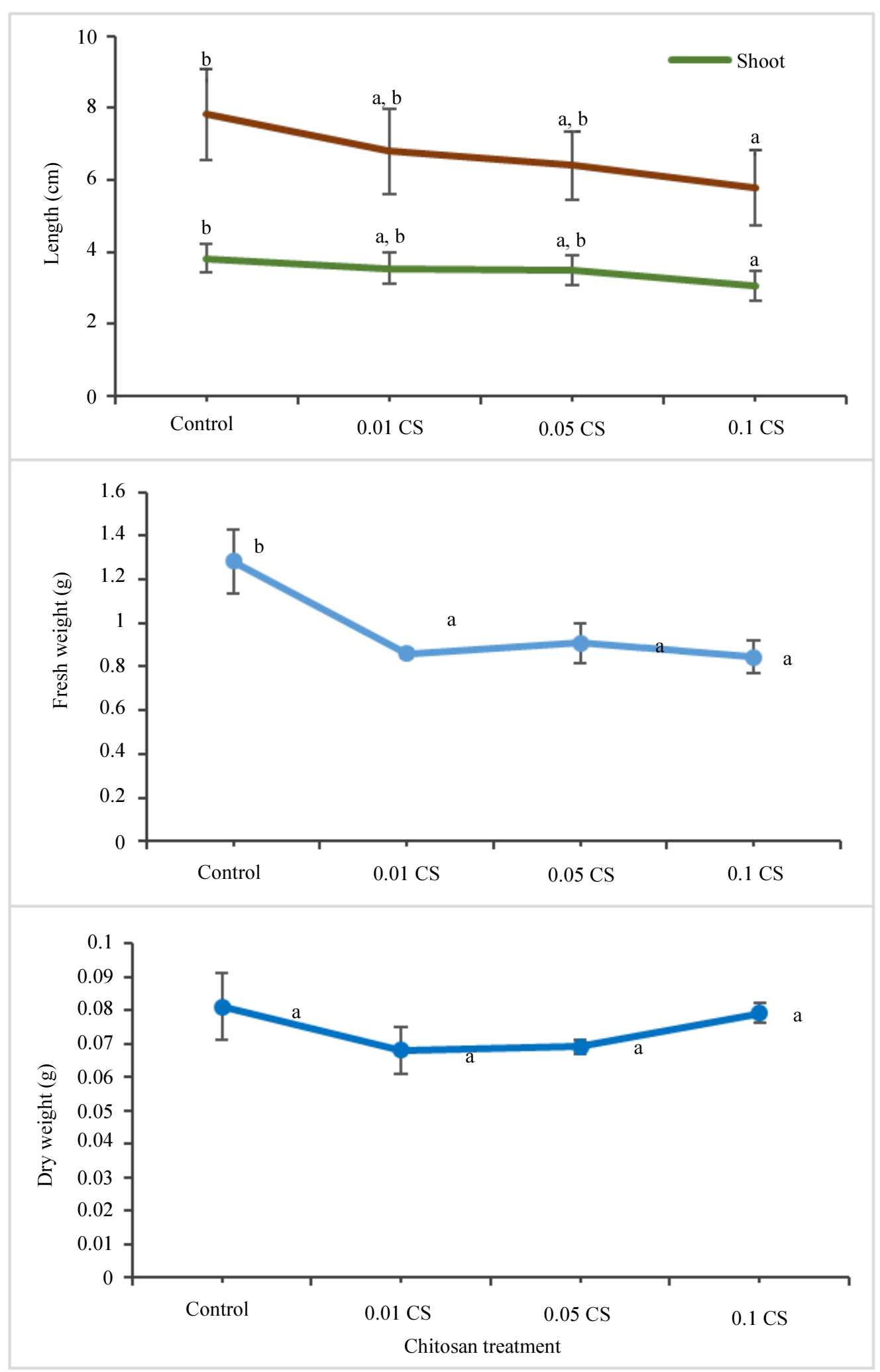

Fig. 1: Plant growth of fenugreek sprouts treated with different concentrations of chitosan. The final chitosan concentrations were $0.1,0.5$ and $1 \mathrm{mg} / \mathrm{mL}(n=3) . \mathrm{R}^{2}=0.9992$ and the bars with different letters are significantly different from each other at $p \leq 0.05$ according to Tukey's Multiple Range Test 


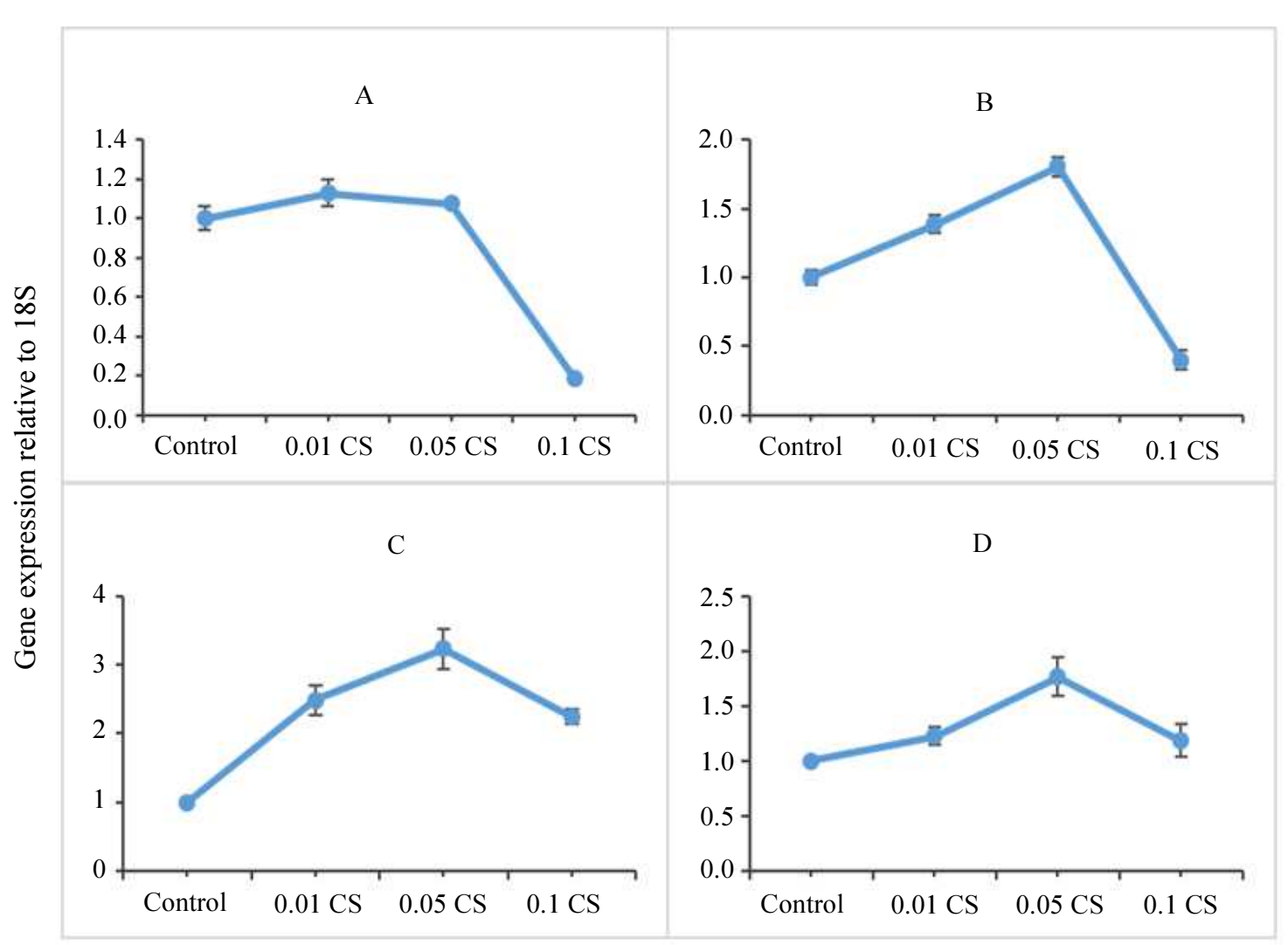

Chitosan

Fig. 2: Expression of diosgenin biosynthetic genes in fenugreek sprouts treated with different chitosan concentrations. The final chitosan concentrations were $0.1,0.5$ and $1 \mathrm{mg} / \mathrm{mL}(n=3)$. Thegraph of (A), TfSQS: Trigonella foenum graecum squalene synthase; (B), TfSQLE: Trigonella foenum graecum squalene monooxygenase; (C), TfCAS Trigonella foenum graecum cycloartenol synthase; and (D), TfSTRL: Trigonella foenum graecum sterol-3- $\beta$-glucosyl transferase

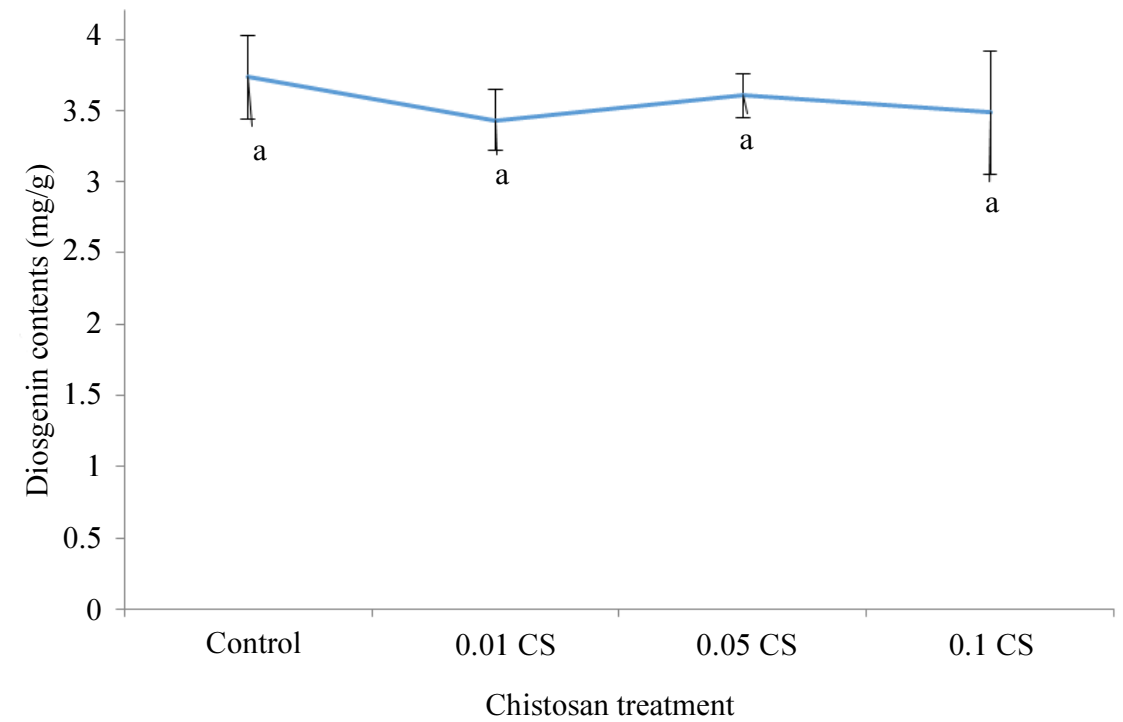

Fig. 3: Diosgenin content (mg/g DW) in chitosan-treated sprouts. The final chitosan concentrations were $0.1,0.5$ and 1 $\mathrm{mg} / \mathrm{mL}(n=3) . \mathrm{R}^{2}=0.9992$ and the bars with different letters are significantly different from each other at $p \leq 0.05$ according to Tukey's Multiple Range Test 


\section{Conclusion}

Diosgenin is the principal precursor compound in the manufacture of several synthetic steroidal drugs. It is acted as a promising bioactive biomolecule exhibiting various biological properties, like hypolipidemic, hypoglycaemic, antioxidant, antiinflammatory and antiproliferative activities. Sprouts of $T$. foenumgraecum treated with elicitor like chitosan responded negatively both for shoot and root growth, but these treatments enhanced the upregulation of diosgenin biosynthetic genes i.e., TfSQS, TfSQLE, TfCAS and TfSTRL. Variations in diosgenin levels remained almsot similar in the chitosan treatment compared to control. Though chitosan did not response well for growth and accumulation of diosgenin in the sprouts of $T$. foenumgraecum but showed upregulation trends of all diosgenin biosynthetic genes. These information might be helpful for future study in this arena.

\section{Acknowledgment}

This study was supported by research fund of Chungnam National University in 2018.

\section{Author's Contributions}

Do Yeon Kwon: Performed the experiments, analyzed the data and prepare the manuscript.

Jae Kwang Kim: Performed the experiments, analyzed the data and prepare the manuscript.

Sang Un Park: Designed the experiments, coordinated the implementation of research work.

\section{Ethics}

This manuscript has not been published or presented elsewhere in part or in entirely and is not under consideration by another journal. All the authors have approved the manuscript and agree with submission to your esteemed journal. There are no conflicts of interest to declare.

\section{References}

Chaudhary, S.K., M.C. Chikara, A. Sharma, B. Chaudhary and P.S. Alam Syed et al., 2015. Elicitation of diosgenin production in Trigonella foenum-graecum (Fenugreek) Seedlings by Methyl Jasmonate. Int. J. Mol. Sci., 16: 29889-99.

Chen, C.T., Z.H. Wang, C.C. Hsu, H.H. Lin and J.H. Chen, 2015. In vivo protective effects of diosgenin against doxorubicin-induced cardiotoxicity. Nutrients, 7: 4938-4954.
Cho, M., H. No and W. Prinyawiwatkul, 2008. Chitosan treatments affect growth and selected quality of sunflower sprouts. J. Food Sci.

Christen, P., 2002. Trigonella species: In vitro culture and production of secondary metabolites. Springer.

El Hadrami, A., L.R. Adam, I. El Hadrami and F. Daayf, 2010. Chitosan in plant protection. Marine Drugs, 8: 968-987.

Ferri, M. and A. Tassoni, 2011. Chitosan as Elicitor of Health Beneficial Secondary Metabolites in in vitro Plant Cell Cultures. In: Handbook of Chitosan Research and Applications, pp: 389-414.

Jesus, A.P.J.M., E. Gallardo and S. Silvestre, 2016. Diosgenin: Recent highlights on pharmacology and analytical methodology. J. Anal. Meth. Chem., 2016: 1-16.

Malerba, M. and R. Cerana, 2016. Chitosan effects on plant systems. Int. J. Mol. Sci., 17: 996-996.

Rahmati-Yamchi, M., S. Ghareghomi, G. Haddadchi, M. Milani and M. Aghazadeh et al., 2014. Fenugreek extract diosgenin and pure diosgenin inhibit the hTERT gene expression in A549 lung cancer cell line. Mol. Biol. Rep., 41: 6247-6252.

Reddy, M.B., J. Arul, P. Angers and L. Couture, 1999. Chitosan treatment of wheat seeds induces resistance to Fusarium graminearum and improves seed quality. J. Agric. Food Chem., 47: 1208-1216.

Salachna, P. and A. Zawadzińska, 2014. Effect of chitosan on plant growth, flowering and corms yield of potted freesia. J. Ecol. Eng., 15: 97-102.

Snehlata, H.S. and D.R. Payal, 2012. Fenugreek (Trigonella foenum-graecum L.): An overview. Int. J. Curr. Pharm. Rev. Res., 2: 169-87.

Uddin, M.R., Y.K. Kim, S.U. Park and J.Y. Pyon, 2010. Enhancing sorgoleone levels in grain sorghum root exudates. J. Chem. Ecol., 36: 914-922.

Walker, R., S. Morris, P. Brown and A. Gracie, 2004. Evaluation of potential for chitosan to enhance plant defense. A Report for the Rural Industries Research and Development Corporation, Australia, RIR DC Publication. 